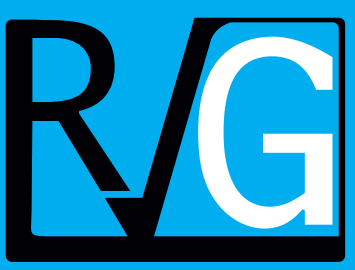

Año 21 No. 74

Abril - Junio 2016

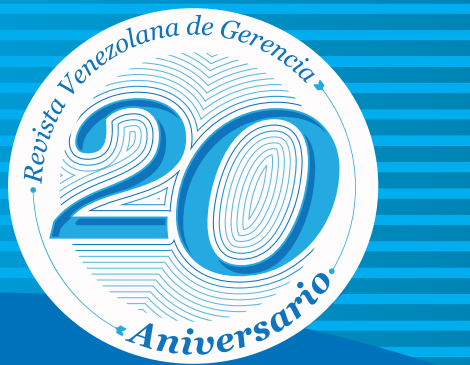

Venezolana de
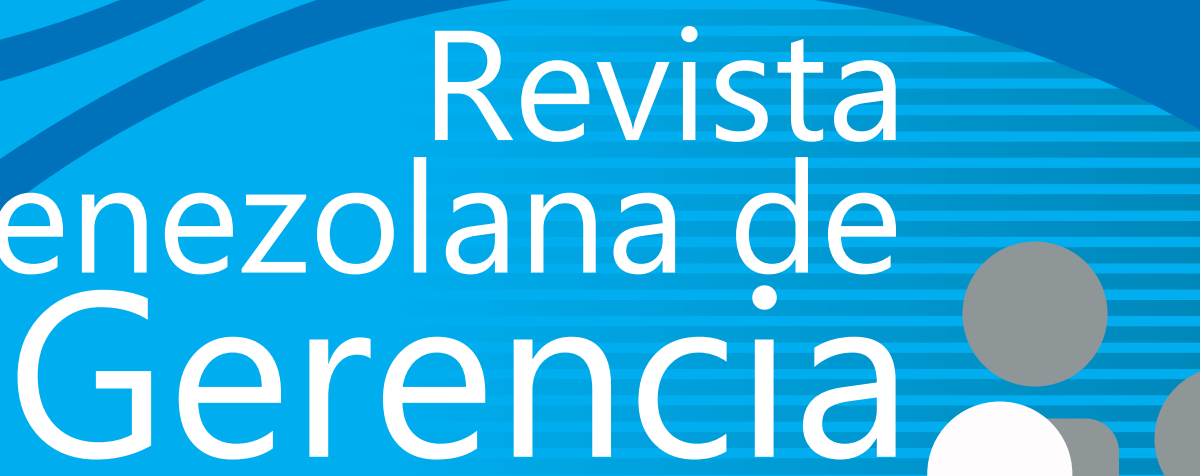


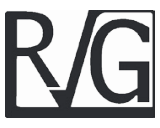

\title{
Elementos determinantes de la ecoinnovación en hotelería de Huatulco, México
}

\author{
Velázquez-Castro, Judith Alejandra ${ }^{1}$ \\ Vargas-Martínez, Elva Esther ${ }^{2}$ \\ Olíver-Espinoza Rubén ${ }^{3}$ \\ Cruz-Jiménez Graciela ${ }^{4}$
}

\section{Resumen}

La ecoinnovación es un término reciente que día a día ha adquirido un importante significado para las empresas y los consumidores; no obstante la literatura sobre ésta en las organizaciones turísticas es escasa. Por lo anterior, este artículo analiza aquellos elementos que determinan la ecoinnovación en los establecimientos de hospedaje del destino turístico sustentable, Bahías de Huatulco, México y su dinámica con respecto al comportamiento ecoinnovador que asumen. La presente investigación emplea el método cuantitativo, y como técnica de recolección de datos la aplicación de entrevistas semiestructuradas a gerentes y administradores de establecimientos con diversas categorías turísticas. Los principales resultados después de analizar los cuatro determinantes: 1) factores específicos de cada empresa; 2) mercado; 3) tecnologías ambientales; y 4) regulación, muestran que la demanda incide en los hoteles para generar innovaciones ambientales, que las inversiones en equipo y tecnología se realizan con la finalidad de disminuir costos y el cumplimiento de la legislación ambiental; y que son pocos los hoteles que relacionan los estándares ecológicos con la posibilidad de lograr una ventaja competitiva en el mercado.

Palabras clave: innovación; ecoinnovación; turismo; hotelería; sustentabilidad.

Recibido: 15.04.16 Aceptado: 30.05.16

1 Estudiante del Doctorado en Estudios Turísticos, Facultad de Turismo y Gastronomía, Universidad Autónoma del Estado de México, email: judithalejandra666@gmail.com (correspondencia).

2 Doctora en Ciencias Ambientales, Profesora Investigadora y Coordinadora del CIETUR, Facultad de Turismo y Gastronomía, Universidad Autónoma del Estado de México.

3 Doctor en Ciencias Sociales, profesor investigador del Centro de Investigaciones Económicas, Administrativas y Sociales (CIECAS), Instituto Politécnico Nacional.

4 Doctora en Ciencias Sociales, profesora investigadora del CIETUR, Facultad de Turismo y Gastronomía, Universidad Autónoma del Estado de México. 


\title{
Determinants of eco-innovation from the hotelier of Huatulco, Mexico
}

\begin{abstract}
Eco-innovation is a recent term that every day has become an important meaning for businesses and consumers; however the literature on this in tourist organizations is limited. Therefore, this article analyzes the elements that determine the eco-innovation in lodging establishments sustainable tourist destination, Huatulco, Mexico and dynamic behavior with respect to ecoinnovation they assume. This research uses quantitative method, and as a data collection technique application of semi-structured interviews managers and managers of establishments with various tourist categories. The main results after analyzing the four determinants: 1) specific factors of each company; 2) market; 3) environmental technologies; and 4) regulation, show that demand affects hotels to generate environmental innovations, investments in equipment and technology are made in order to reduce costs and compliance with environmental legislation; and that few hotels ecological standards relating to the possibility of achieving a competitive advantage in the market.t
\end{abstract}

Keywords: innovation; eco-innovation; tourism; hospitality; sustainability.

\section{Introducción}

Las empresas innovan, -el origen etimológico de innovación proviene del prefijo in, introducir o estar en; y del latín novus, nuevo; es decir, la introducción de algo nuevo-a través de una amplia gama de actividades, que incluyen la adquisición de nuevos productos, procesos y tecnologías, la combinación de los ya existentes dentro de la organización, o por el conocimiento de nuevas formas de gestión, entre otras. Así, ante el panorama de competitividad que se vive en la actualidad y la crisis ecológica que agobia al mundo, las empresas han tenido que impulsar una serie de cambios que atiendan por una parte su rentabilidad; y por otra, mejore su desempeño organizacional para proteger la naturaleza.

En la pretensión por adoptar nuevas formas de producción, aparece el término de ecoinnovación, cuyo fin se asienta en la reducción de los riesgos ambientales, la contaminación y otros impactos negativos a los recursos naturales. No obstante, Horbach et al, (2012) señalan que la ecoinnovación puede ser el resultado de otras innovaciones, que no necesariamente han sido motivadas por las preocupaciones ambientales, sino por razones tan diferentes a lo ecológico; como el aumento de cuotas de mercado o la reducción de los costos de operación. Así entonces, son las mismas empresas quienes ponen en práctica estrategias ambientales para incrementar sus beneficios económicos, tales como: satisfacer las necesidades de los clientes, mejorar la reputación de la empresa ante la opinión pública o blindar a la organización contra multas y sanciones por parte de la autoridad ambiental.

Por lo que, este artículo analiza aquellos elementos que determinan la ecoinnovación en los establecimientos de hospedaje del destino turístico sustentable, Bahías de Huatulco, México, registrados en la Asociación de Hoteles y Moteles 
de Huatulco, A.C. De esta manera, la importancia de identificar los determinantes de ecoinnovación en empresas de servicios, radica en proporcionar apoyo para facilitar la aplicación y actualización de información, referente a las nuevas tendencias tecnológicas ambientales y de gestión que intervienen en las prácticas ecológicas bajo la naturaleza específica de operación en éstas.

Asimismo se presenta la discusión teórica de la ecoinnovación, como soporte a la praxis en los establecimientos de hospedaje. En seguida, se exponen los determinantes que inciden en las empresas para el implemento de ecoinnovaciones, así como su tipología, fases y estrategias; hasta llegar a la taxonomía o clasificación de las empresas ecoinnovadoras. También se aborda el marco contextual de la comunidad turística de Bahías de Huatulco, México; con el cual se pretende exponer la situación actual de los establecimientos de hospedaje en este destino turístico.

Se exponen como principales resultados las características y los factores que impulsan las prácticas de ecoinnovación en los establecimientos de hospedaje. Cabe resaltar que, las veinte empresas participantes en la investigación se clasificaron en tres grupos de innovación ambiental mutuamente excluyentes. El primero está formado por empresas Estratégico eco-adopters, que manifiestan haber adoptado nuevas estrategias de gestión y marketing o comprado productos o tecnologías verdes a otras empresas. El segundo grupo está conformado por empresas Pasivo- ecoinnovadoras, las cuales fueron más propensas a innovar sin incluir explícitamente estrategias de innovación. En el tercer grupo se incluye a las empresas No ecoinnovadoras, que de acuerdo al análisis, son empresas con un bajo o nulo nivel de capacidades innovadoras; entre sus particularidades resalta su pequeño tamaño, la ausencia de personal preparado profesionalmente, y la falta de inversión en productos o tecnologías ambientales.

\section{El marco teórico de la ecoinnovación}

A continuación se expone la revisión literaria sobre el tema de estudio, la cual es el soporte para definir el problema de investigación y construir el marco teórico para el diseño de los métodos y procedimientos para cumplir con el objetivo planteado. La revisión de la literatura da lugar a la identificación de los modelos teóricos y conceptuales sobre el tema de ecoinnovación que orientan la investigación.

\subsection{Naturaleza del concepto}

La acepción de innovación ha ido evolucionando en paralelo con los cambios en la economía mundial y la globalización. De este modo y debido a los avances en materia de ciencia y tecnología, así como en materia de sustentabilidad ${ }^{3}$, aparece el término de ecoinnovación. Sin embargo, es un concepto que sigue desarrollándose bajo las premisas de sustentabilidad, para comprender cómo los actores involucrados en el proceso de innovación puede afectar o influir en su entendimiento.

3 En el año de 1987, la Comisión Mundial sobre Medio Ambiente y el Desarrollo (CMMAD) propone la idea de la sustentabilidad, diciendo que una sociedad sustentable es aquella que: satisface las necesidades del presente sin comprometer la capacidad de las generaciones futuras para satisfacer sus propias necesidades. 
De este modo, el término de ecoinnovación surge desde finales del siglo pasado, como respuesta a la serie de problemas -como deterioro o pérdida total de flora y fauna, y degradación del suelo, entre otras- causados por el uso irracional de los recursos naturales, y de los daños ocasionados al ambiente -como lo es la contaminación atmosférica o del agua-por la producción excesiva de algunos bines y por el uso de tecnologías que repercuten negativamente en el ambiente.

La ecoinnovación puede vislumbrarse desde distintas posturas. Desde la perspectiva sustentable, la ecoinnovación debe reducir el consumo de energía y recursos hídricos, uso del espacio -ecodiseño- y preservar la biodiversidad (Hellström, 2006). Desde el punto de vista económico, es entendida como una estrategia para lograr un crecimiento económico y contribuir al progreso de los países, mediante cambios en los patrones de producción y de consumo, sustentados en la productividad y conseguir reducir significativamente el impacto en el ambiente y la contaminación a lo largo de la cadena de valor (Rennings, 2000). La ecoinnovación contribuye a mejorar significativamente las actividades comerciales en un contexto de mercados abiertos, competitivos y adecuadamente regulados (OECD, 2011).

Desde la perspectiva empresarial, la ecoinnovación es concebida como la producción, creación, aplicación o explotación de un bien, servicio, proceso productivo o sistema de gestión competitivo que es nuevo para la empresa, cuyo resultado es, durante su ciclo de vida, reducir la contaminación, los riesgos ambientales y otros impactos negativos asociados al uso de recursos en comparación con alternativas más eficientes y satisfacer así las necesidades humanas proporcionando una mejor calidad de vida (Measuring Eco-innovation Project, 2007; Reid y Miedzinski, 2008; Comisión Europea para el Medioambiente, 2009; Kemp y Pearson en Miret et al, 2011; OECD, 2011).

En este argumento, las empresas tienen la oportunidad de poner en marcha distintas estrategias que resulten en una ventaja competitiva. Esto es, de un predominio en relación a un mejor servicio -sustentable-respecto al de la competencia. Las actividades de innovación en cualquier empresa -sin importar su giro-, deben encaminarse al propósito de mejorar o ampliar las oportunidades de supervivencia o crecimiento a largo plazo, tomando en cuenta la importancia y rol -fundamentalde los recursos naturales.

Desde el enfoque sustentable, la ecoinnovación hace referencia a la modificación de los patrones de consumo, pasando del consumismo al consumo eficiente que promueve la reutilización de recursos, valorización de residuos, y, en general, la optimización del potencial productivo de los recursos disponibles (Centro de Investigación de Recursos y Consumos Energéticos, 2013; Valero, 2013). Por tanto, la ecoinnovación promueve el desarrollo sustentable a través de modelos productivos que permiten el desarrollo de la sociedad, sin perjuicio a la naturaleza y como fuerza impulsora para el futuro.

Así entonces, la ecoinnovación no se limita al diseño o consumo de productos $y$ servicios ambientales, o aquellas innovaciones que hayan tenido una motivación exclusivamente ambiental, sino también al incremento de valor agregado a los clientes y para las empresas, a través de la búsqueda de nuevas soluciones para los problemas existentes.

Al igual que la innovación, la ecoinnovación puede ser tecnológica, organizacional, intangible o sistémica, 
$y$, como toda innovación, requiere de conocimientos, atención, de capacidades empresariales, de recursos -humanos, materiales, económicos- y de la coordinación entre diversos actores para su desarrollo y adopción (Kemp y Oltra, 2011).

Tanto autores como organismos internacionales (Kemp y Pearson, 2008; Comisión Europea para el Medioambiente, 2009; Kemp y Oltra, 2011; OECD, 2011), hacen énfasis en el uso y desarrollo de innovaciones tecnológicas para crear soluciones sustentables, que permitan a las empresas un mejor uso de los recursos naturales y que reduzcan las repercusiones negativas del crecimiento económico sobre la naturaleza.

Por último, la ecoinnovación puede fomentar e impulsar dentro de la empresa, un cambio en sus actividades, es decir, una modificación de rutinas y procesos, que contribuyan al desarrollo de servicios turísticos sustentable; favoreciendo a la vez, tanto su compromiso con la sociedad y garantizando la protección del ambiente, sin dejar de mencionar el beneficio económico que puede obtener por el ahorro de costos y el uso eficiente de sus recursos.

La ecoinnovación también hace referencia a la entrega de productos y servicios -con precios competitivos-, que satisfacen las necesidades humanas $y$ favorecen la calidad de vida, mientras que reducen en forma progresiva los impactos ecológicos y la intensidad de recursos a través del ciclo de vida, hasta un nivel en equilibrio con los límites de cambio aceptable de la tierra, es decir, crear más valor con el menor impacto posible.

Por otro lado, también responde con mayor eficacia a las apremiantes exigencias del desarrollo sustentable, al tiempo que ayuda a fomentar la competitividad de las empresas basándose e influyendo -al mismo tiempoen la estructura social. Supone además, la introducción de mejoras ambientales radicales en los productos, las tecnologías o la organización empresarial, va más allá de las mejoras incrementales contempladas por la ecoeficiencia (Carrillo et al, 2009; Fundación Canaria Medioambiental, 2011).

Así entonces, la ecoinnovación contribuye para avanzar hacia la sustentabilidad mediante el desarrollo de innovaciones fundadas en la estructura social, encaminadas al cuidado y uso eficiente de los recursos naturales (ecoeficiencia) que reduzcan las repercusiones negativas de las actividades empresariales sobre el ambiente. Además, no sólo procura el cuidado de la naturaleza, sino también promueve ventajas competitivas para las empresas.

\subsection{Determinantes de la ecoinnovación}

Para cualquier empresa, la innovación representa una ventaja competitiva para diferenciarse en su mercado; así, puede intuirse que las empresas se ven influenciadas $u$ obligadas -en algunas ocasiones- a innovar por cuatro principales razones:

En primer lugar, el mercado es un factor que afecta la actividad innovadora de cualquier empresa de diversas maneras, ya que contribuye al desarrollo de nuevos servicios, dado que las empresas modifican los servicios ya existentes para aumentar sus ventas, minimizar los costos o mejorar el posicionamiento en el mercado, y con frecuencia este factor puede ser el motor de la innovación. En los últimos años, las empresas han migrado a procedimientos basados en la protección del ambiente, por lo que han vislumbrado el potencial económico de ofertar 
servicios respetuosos con la naturaleza a consumidores que adoptan pautas de compra ambientalmente responsables, con el fin de reducir su impacto sobre la naturaleza.

Horbach et al, (2012) advierten que existe evidencia empírica de que los beneficios esperados por los clientes desempeñan un papel clave para las innovaciones ecológicas, en cuanto éstos obtengan un valor añadido a partir de servicios o productos diferenciados, sin descuidar la satisfacción de sus necesidades. Así que, las empresas tienen que demostrar que son promotoras de un comportamiento compatible con la sustentabilidad.

Entre los factores que determinan la influencia del mercado sobre la ecoinnovación, se tiene a) la accesibilidad al mercado, incluyendo las posibilidades de establecer relaciones estrechas con los clientes, así como la dimensión del mercado y la facilidad de acceso a éste; b) la estructura empresarial $y$ el entorno competitivo, que tiene relación con proveedores competitivos y comprometidos, al igual que la empresa con el cuidado ambiental; c) el desarrollo de nuevos métodos de comercialización y venta, que son los métodos utilizados para vender servicios a los clientes; y d) nuevos métodos de promoción, los cuales implican la utilización de nuevos conceptos para promocionar los servicios.

En cuanto a los factores específicos de cada empresa, éstos hacen referencia a la estrategia empresarial, la reducción de costos, el conocimiento de mecanismos de transferencia de tecnología y la participación en redes, entre otras. Desde esta perspectiva, las capacidades organizacionales juegan un papel importante (Kammerer, 2009; Wagner 2009). Por ejemplo: el compromiso de la alta dirección, el trabajo en equipo, la capacitación de los empleados, y el uso de técnicas como el mapeo de procesos o el análisis de causa efecto, con la finalidad de conducir a la organización por el camino del cuidado ambiental.

El tercer determinante, se vincula con el uso de tecnologías o de avances tecnológicos, que tienen implicación en la calidad en el producto, con el objetivo de fomentar el ahorro y la eficiencia energética, la eficiencia de los materiales y/o la gama de productos existentes en el mercado, mediante la implantación de ecotecnologías -también conocidas como tecnologías verdes-, sin embargo, éstas suelen ser las que implican mayores costos para las empresas. El término de ecotecnología, hace alusión a las tecnologías que tienen como objetivo preservar el ambiente, ello, mediante alternativas como el uso de recursos renovables o de utilizar la energía de manera más eficiente, a fin de aminorar los residuos que causan la contaminación de los ecosistemas y la atmósfera.

Finalmente, la regulación se integra por la política ambiental, los estándares ambientales, los instrumentos de regulación (responsabilidad ambiental, impuestos verdes) y las ecoetiquetas. La regulación gubernamental es importante para impulsar a las empresas a reducir la contaminación y las emisiones de agua o ruido; asimismo sirven para evitar el uso de sustancias peligrosas y aumentar el reciclaje de productos. Estos instrumentos permiten a las empresas valerse de su desempeño y/o compromiso ambiental, en su estrategia de comercialización o en las negociaciones con el Gobierno.

\subsection{Taxonomía de las empresas ecoinnovadoras}

La elección de innovar a través de la adquisición de productos, servicios 
o tecnologías o a través del diseño de procesos u otras actividades dependerá de las necesidades y características propias de la empresa. Así, las empresas pueden optar por crear o comprar una innovación -ya sea a través del uso de servicios de consultoría o mediante la contratación de nuevos empleados-; por lo que pueden elegir entre diferentes opciones. Así, las empresas pueden ejercer diversas prácticas de ecoinnovación, pero no necesariamente para disminuir o contrarrestar los daños ambientales que las llevarán hacia un mejor comportamiento ambiental, sino motivadas por sus propios intereses. Bajo ese argumento, Kemp y Pontoglio (2007), explican en cuatro grupos la taxonomía de las empresas ecoinnovadoras, de acuerdo a su posición frente a la creación o adopción -o no- de ecoinnovaciones. Los autores ubican en el primer grupo a las empresas Estratégico ecoinnovadoras, que se caracterizan por ser activas en el diseño y producción de ecotecnologías u otro tipo de innovaciones ecológicas para su venta a otras empresas; además de ser las únicas que llevan a cabo actividades de I+D.

En el segundo grupo, se encuentran las empresas Estratégico eco-adopters, que se caracterizan por aplicar voluntariamente las innovaciones ecológicas, tanto de desarrollo, diseño propio o adquirido de otras empresas. Asimismo, cuentan con una estrategia específica para la integración de actividades de innovación a sus prácticas habituales.

El tercer grupo, es de las Pasivasecoinnovadoras, que son las empresas que implementan ecoinnovaciones en procesos, de organización, productos, etc., que pueden resultar en beneficios económicos y ambientales; pero a diferencia de los eco-adopters, no cuentan con una estrategia específica definida de ecoinnovación dentro sus procesos. Es decir, que poseen un nivel moderado de capacidades innovadoras, ya que las actividades de ecoinnovación que realizan no son intencionales, por lo que pueden tener un fin distinto al relacionado con la conservación o cuidado ambiental, pero que en última instancia sí favorece la mejora ambiental. El cuarto y último grupo está conformado por las empresas No-ecoinnovadoras, en donde no existen actividades específicas de ecoinnovación.

Esta tipología hace visible que las categorías identificadas son integrales y suficientemente precisas, además de ser excluyentes entre sí. Lo anterior se debe a que la ecoinnovación pasiva puede interpretarse como la falta de una estrategia ambiental específica para llevar a cabo actividades de innovación, en función de las actividades que podrían estar activas en las categorías de las empresas denominadas estratégico ecoadopters.

Por otro lado, Pearson (2007) sostiene que esta clasificación es útil para concebir nuevas ideas, por la razón que se ve en el tema la intencionalidad -decisión por innovar-, es decir, en la adopción y diseño de innovaciones ecológicas específicas; por lo que también es posible medir los tipos de ecoinnovación sí una empresa las está adoptando.

Finalmente, cabe resaltar que la capacidad de innovación de la empresa es precisa para participar en cualquier tipo de actividades encaminadas a innovar; es decir, en la introducción u organización de nuevos o mejorados procesos, productos, herramientas de gestión o marketing. Esta capacidad de innovación, es un factor importante que incide en el beneficio de la empresa, y 
los establecimientos de hospedaje de Bahías de Huatulco no son la excepción, pues a través de ella, los gerentes pueden idear soluciones nuevas a los problemas o desafíos a los que se enfrenta el negocio; además, de considerar otros factores que también determinan y proveen la base de la supervivencia y éxito de su futuro.

\section{Bahías de Huatulco, un destino turístico sustentable}

El Centro Integralmente Planeado (CIP) de Bahías de Huatulco, se ha caracterizado por ostentar una imagen de protección y conservación ambiental, ya que desde sus inicios - en la década de los 80- se priorizó el cuidado de los recursos naturales, al declararlo como reserva natural protegida. De acuerdo con datos de FONATUR (2012), en Huatulco 6.35\% de la superficie total está destinada a la construcción de la zona turística, 3.45\% es para zona urbana y $90.19 \%$ posee el carácter de reserva ecológica.

Al día de hoy, Huatulco cuenta con una infraestructura turística capaz de satisfacer las necesidades de cualquier turista, integrada por un aeropuerto internacional, un embarcadero, hoteles, restaurantes, clubes nocturnos y campo de golf. La oferta de hospedaje se compone por 24 establecimientos, de los cuales $40 \%$ pertenecen a la categoría de Gran turismo, Lujo y Especial; $25 \%$ a la categoría de cuatro estrellas; $25 \%$ a la categoría de tres estrellas y $10 \%$ a la de dos estrellas (Asociación de Hoteles y Moteles de Huatulco, 2016).

EI CIP cuenta con seis plantas de tratamiento de aguas residuales -y otras dos plantas más preautorizadasque abastecen a toda la Comunidad. La energía eléctrica que ofrece es suministrada por el parque de "La Ventosa" ubicado en el Istmo de Tehuantepec, Oaxaca y es en $90 \%$ eólica y 10\% hidroeléctrica (Equipo Verde Huatulco, 2014).

Desde hace poco más de una década, los distintos actores que forman parte de esta comunidad turística, llevan a cabo labores para la sustentabilidad del lugar. Entre los logros alcanzados, se encuentra: la certificación de Earthcheck gold, que consiste en implementar programas y acciones permanentes de capacitación y sensibilización ambiental, conservación de sus recursos naturales, así como el mejoramiento de la infraestructura de servicios (EVH, 2016), para mejorar el desempeño operacional de las empresas prestadoras de servicios turísticos que convierte a Huatulco en la primer Comunidad Turística Sustentable en el continente americano y la tercera en el mundo; el Distintivo $S$, otorgado a las empresas turísticas, por la Secretaría de Turismo (SECTUR), que reconoce las buenas prácticas en el desarrollo de proyectos turísticos y el compromiso de las empresas mexicanas que operan bajo los más altos estándares ambientales; el sello Bandera Azul para la playa de Chahué, ya que cumple con las normas de calidad del agua, la seguridad, la prestación de servicios generales y de ordenación del medio ambiente; por último, cuenta con la designación australiana de Carbon Low Zone, que consiste en la reducción de la huella de carbono.

No obstante, en un intento por ampliar la oferta de cuartos, actualmente se construye una subestación de energía eléctrica -para abastecer a los nuevos hoteles-, un corredor turístico comercial y próximamente otro campo de golf; mismos que ponen en riesgo el futuro sustentable de este destino. En 
ese sentido, los esfuerzos propuestos y emprendidos, hasta el momento, por lograr posicionar a Huatulco como destino turístico de calidad internacional, pueden no lograr una consolidación, que deje rezagados o minimizados los aspectos ambientales y sociales, enfatizando los beneficios económicos de las actividades turísticas.

\section{Acercamiento metodológico al caso de estudio}

De acuerdo con Horbach, Rammery Reanings (2012) existen cuatro determinantes de la ecoinnovación en turismo, mismos que fueron comparados con los resultados del estudio empírico: a) el mercado; b) los factores específicos de cada empresa; c) las tecnologías ambientales; y d) la regulación. En este análisis, fueron consideradas las cuatro dimensiones, así como los indicadores asociados que determinan el comportamiento ecoinnovador de una empresa. Con base en ello, se diseñaron dos instrumentos de investigación, mismos que representan una fuente única de información para conocer y explorar sobre las distintas actividades y determinantes de ecoinnovación en los hoteles de Huatulco.

La investigación tuvo aplicación práctica en el año 2014, con la colaboración de 20 directivos de empresas de hospedaje registradas ante la Asociación de Hoteles y Moteles de Huatulco, A.C., lo que representó una tasa de participación del $83 \%$ de la población objeto de estudio.

Se parte del hecho de que la disposición por innovar es una decisión estratégica que, depende de las acciones tomadas por la gerencia, con el objetivo de mejorar la competitividad de la empresa. Asimismo, se considera que la percepción de los gerentes influye en la decisión por innovar o no, y de cómo favorece la ecoinnovación a la organización. Finalmente, tomando como base la fundamentación teórica y el estudio empírico, se procede con la identificación y análisis de los resultados obtenidos.

\section{Evaluación de resultados de ecoinnovación}

En una primera aproximación, la evidencia empírica pone de manifiesto que las organizaciones de mayor tamaño o las pertenecientes a una cada hotelera, estimulan la innovación a través de factores internos específicos, como habilidades, proyectos o una cultura creativa; en donde las personas tienen la posibilidad de proponer nuevas soluciones en áreas de oportunidad identificadas o a problemas detectados. Así, se parte de la idea de que la capacidad de innovación es la base sobre de una organización ecoinnovadora.

De acuerdo con la taxonomía de las empresas ecoinnovadoras, y para efectos de esta investigación -dada la naturaleza de los establecimientos de hospedaje- se toman en cuenta sólo tres de los cuatro grupos propuestos por Kemp y Pontoglio: 1) estratégico eco-adopters, 2) Pasivo-ecoinnovadores y 3) Noecoinnovadores; exceptuando al grupo de los estratégico-ecoinnovadores, que son el grupo que lleva a cabo actividades de I+D. Así, se logran identificar los tres grupos en los 20 establecimientos de hospedaje (ver gráfico 1 ). 


\section{Grafico 1 \\ Taxonomía de las empresas ecoinnovadoras de Huatulco}

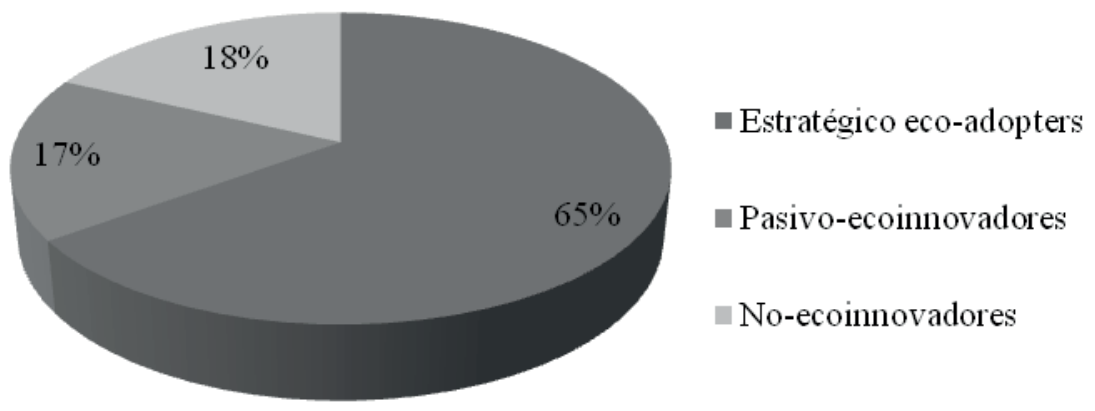

Fuente: elaboración propia con base en Kemp y Pontoglio (2007).

En el gráfico anterior, se observa que el $65 \%$ del total de hoteles analizados, son Estratégico eco-adopters; este grupo está conformado por los hoteles pertenecientes a una cadena hotelera -nacional o internacional- y de mayor tamaño. Lo anterior se debe, a que la mayoría de las innovaciones ambientales requiere de grandes inversiones, sobre todo las relacionadas con el área de ecotecnologías, que es el área en donde se realiza una mayor inversión, sobre todo en tecnologías para el ahorro del consumo de agua y electricidad.

Por otra parte, los factores tecnológicos son importantes porque coadyuvan a las empresas a mejorar las posibilidades de prestación del servicio, además de que son condiciones necesarias para que adquieran ventajas competitivas. Conjuntamente, estas empresas se caracterizan por integrar estrategias de ecoinnovación, como el uso de productos y tecnologías ambientales, que les permite ahorrar costos por la disminución de consumo de materiales y energía, por el uso de aparatos eléctricos eficientes o de energías alternativas; y les facilitael ingreso a nuevos mercados y/o el incremento de su participación en el mismo.

Entre los cambios que han adoptado, resalta la adquisición de reductores de flujos de agua en regaderas, fregaderos, inodoros y lavabos, riego de jardines por sistema de aspersión, encendido automático de luces en áreas públicas o el uso de lámparas solares y de LED. Asimismo, la adquisición de alimentos orgánicos -que no utilizan ningún tipo de pesticida o plaguicida- $y$ el uso de productos amigables con el ambiente a pesar de su alto costo, tales como productos de limpieza o cuidado personal, les permite proyectar una mejor imagen.

En cuanto a estrategias de ecoinnovación se encuentra presente el ecodiseño, sobre todo en hoteles nuevos o renovados que han optado por el concepto de la construcción ecológica, teniendo como resultado el posterior ahorro de energía, recursos naturales y económicos. Actualmente, existen 
hoteles que cuentan con instalaciones modernas, en donde destacan las áreas que aprovechan eficientemente la luz solar, infraestructura para la captación y almacenamiento de agua de lluvia y el manejo de flora endémica para reducir el consumo de agua potable a través de la disminución de la necesidad de riego.

Otro de los motivos, por el cual los establecimientos de hospedaje han optado por lo "ecológico", es decir, por instituir una serie de normas empresariales que favorecen la gestión ambiental responsable, surge por el marco normativo y por un sistema voluntario que favorece e influencia a las partes interesadas, es decir, a los clientes, inversionistas, empleados, grupos ecologistas (EVH) y funcionarios del gobierno. Lo anterior, porque este grupo de hoteles se ve favorecido por la imagen que conciben sus clientes y la sociedad.

Los grandes establecimientos y de mayor categoría son los que tienen más alta ecoeficiencia; sin embargo, sus intereses en la praxis, están orientados por fines económicos y comerciales. Esto se debe a la falta de conocimiento e investigación sobre innovación y desarrollo de productos y servicios a largo plazo, que los limita en el diseño de estrategias para innovar ambientalmente.

Por otro lado, el porcentaje de hoteles Pasivo-ecoinnovadores (17\%), es similar al del grupo de los Noecoinnovadores (18\%). Ambos grupos se caracterizan porque integran a los hoteles de menor categoría (35\% de los hoteles restantes). De acuerdo con el análisis de resultados, el principal obstáculo al que se enfrentan estos grupos, es la falta de capital financiero para invertir en productos o tecnologías menos contaminantes.
Los resultados muestran que, los hoteles Pasivo-ecoinnovadores no cuentan con una estrategia formal de ecoinnovación, además de que no relacionan los estándares ambientales con la posibilidad de mejorar de su imagen y lograr una ventaja competitiva en el mercado, o de realmente generar un cambio proambiental; ya que los hoteles de este grupo, hacen uso de distintas ecoinnovaciones, esperando únicamente obtener beneficios económicos por la reducción de costos. Además suponen que las inversiones en tecnologías verdes, son elevadas.

No obstante, estas empresas han optado por alternativas que les permiten mitigar -indirectamente- los daños que causan al ambiente, como la separación de basura, manejo de residuos, capacitación y sensibilización de su personal, para trabajar dentro de la organización en la concepción de pautas normativas y de mejores prácticas ambientales. Conjuntamente, la realización de reuniones periódicas con el EVH para la mejora de prácticas ambientales que favorezca la continuidad de la certificación de Huatulco como destino turístico sustentable, forma parte de las actividades de este grupo.

$45 \%$ de los administradores de los hoteles Pasivo-ecoinnovadores, señalan que en los últimos años su organización ha comenzado a vislumbrar un mayor potencial en la colaboración con la comunidad, el gobierno y las ONGs; lo que contribuye al desarrollo de programas integrales de reducción del impacto ambiental. Estas actividades las comunican a sus huéspedes, para mejorar su imagen pública, prestigio y responsabilidad sustentable.

Las empresas del tercer y último grupo (No-ecoinnovadores), consideran innecesaria o irrelevante la inversión y 
el diseño de ecoinnovaciones, ya que los precios de su servicio no reflejan los costos en los que podrían incidir (tales como los costos de productos de limpieza $y$ aseo personal $u$ otros materiales amigables con la naturaleza), por lo que no cuentan con una estrategia definida para la generación de ecoinnovaciones.

Asimismo, se evidencia que la regulación y las normas pueden inhibir sus procesos de ecoinnovación, al ser confusas o demasiado específicas; por otro lado, la legislación no incentiva el uso de tecnologías o productos ambientales. Ejemplo de ello, es la ley de residuos sólidos de Huatulco -que ejerce mayor presión sobre las empresas para que éstas incrementen sus iniciativas de conservación y reducción del impacto ambiental-, ya que para los pequeños hoteles esta normativa ha representado un desafío, pues la recolección de residuos se hace a través de la programación por días, de basura orgánica e inorgánica, lo que complica el almacenamiento de los residuos perecederos al no contar con cámaras de refrigeración o con algún tipo de transporte que les permita trasladar dichos residuos.

En resumen, el análisis de los resultados muestra que las empresas con un bajo nivel de capacidades innovadoras, son las de tamaño pequeño, ya que careen de experiencia y de personal preparado profesionalmente, y son las que no invierten en productos o tecnologías verdes. Siendo más propensas a innovar las empresas de mayor tamaño, que son las que adoptan o adquieren ecoinnovaciones. Es importante mencionar que, estos resultados son consistentes para las empresas de hospedaje, pertenecientes al sector servicios. Sin embargo, también hay muchas diferencias en los factores que influyen en los determinantes de la innovación empleados según el tamaño de la empresa. Por ejemplo, las tecnologías, el ecodiseño, los proveedores y los clientes son una fuente importante para los estratégico eco-adopters y para los Pasivoecoinnovadores, pero no para los Noecoinnovadores.

Finalmente, esta capacidad de adaptación a las nuevas necesidades, demuestra realmente el tipo de flexibilidad que solo una alianza sólida, basada en las metas compartidas y la confianza mutua, puede ofrecer. Cabe destacar el papel del Equipo Verde Huatulco (EVH, 2016), que es un grupo ciudadano que integra y organiza a los distintos actores sociales del desarrollo turístico, generando sinergias que permitan definir de manera conjunta y estratégica un camino hacia la sustentabilidad, y el cual ha sido un factor clave para todos los establecimientos de hospedaje, ya que asesora a la comunidad empresarial para la prevención de riesgos y el aprovechamiento de oportunidades relacionadas con las tres esferas de la sustentabilidad, consiguiendo ser un punto de conexión entre el sector empresarial, el gobierno, las universidades y la sociedad.

\section{Conclusiones}

Esta investigación aporta evidencia empírica sobre el tema de ecoinnovación en el sector turístico mexicano, específicamente en la hotelería, ya que actualmente el tema ha sido escasamente abordado entre la literatura del campo disciplinar. Asimismo, contribuye en la identificación de los factores que influyen en los establecimientos de hospedaje, para 
la implementación o desarrollo de ecoinnovaciones.

La ecoinnovación o innovación ambiental se vislumbra como una estrategia eficaz para que las empresas del sector turístico contribuyan a la conservación de los recursos naturales, y al mismo tiempo puedan obtener beneficios económicos por la reducción de costos, al usar energías alternativas -solares y eólicas principalmente-, por la implementación de tecnologías verdes o por la inclusión de nuevas formas de gestión a sus prácticas tradicionales.

Cabe resaltar, que las variables y dimensiones apropiadas fueron seleccionadas a partir del análisis de la literatura, para medir directamente la ecoinnovación en los establecimientos de hospedaje. El principal problema al que se enfrentó esta investigación, fue la falta de indicadores y estadísticas en este campo de estudio. El desafío fue alinear dos grupos de indicadores bien definidos: los indicadores de innovación y los indicadores ambientales, ya que los métodos y las perspectivas para la medición y el uso de indicadores de innovación son diferentes a los indicadores ambientales. La definición de indicadores de ecoinnovación, fue una tarea compleja, sin embargo, se consigue proponer un enfoque para combinar la información de ambos campos de estudio.

En cuanto los resultados analizados, se demuestra que la implementación de innovaciones ambientales en los establecimientos de hospedaje es una alternativa viable para apoyar a la sustentabilidad de los destinos turísticos. La evidencia empírica demostró que, la innovación ambiental contiene elementos y procesos fundamentales, independientemente del sector o empresa de que se trate; y que es una inversión a largo plazo, diseñada para aumentar el valor para el consumidor y al mismo tiempo disminuir los costos en los que incide la empresa durante el proceso de prestación del servicio.

Tal y como se expone en los resultados arrojados por las entrevistas, las empresas hoteleras de Huatulco están adoptando nuevos enfoques de gestión, por diversos motivos. Sin embargo, la rentabilidad es la principal impulsora de la implementación de ecoinnovaciones, quedando en último lugar la ampliación de la gama de bienes y servicios. Asimismo, la evidencia demuestra que sí existen prácticas de ecoinnovación, pero en su mayoría no existen procesos definidos de innovación en las empresas hoteleras, es decir, que hay una falta de sistematización de las actividades relacionadas con dichos procesos.

Se manifiesta que los establecimientos de hospedaje que desarrollan o implementan estrategias ecoinnovadoras, son los pertenecientes a una cadena hotelera y/o los de mayor categoría; siendo la ecoinnovación una actividad derivada del elevado consumo y costo de recursos (energía, productos, etc.) en los que incurren.

Finalmente, este trabajo abre nuevas líneas de investigación, relacionados con la Vigilancia Tecnológica y la Inteligencia Competitiva, que también son temas explorados por otras industrias y con gran potencial para el turismo. Los estudios futuros pueden ampliar la investigación de la VT y IC a través de un estudio empírico en los establecimientos de hospedaje, dado el potencial de esta área de investigación y de su estrecha relación con la competitividad de la industria turística. 


\section{Referencias bibliográficas}

Asociación de Hoteles y Moteles de Huatulco, A. C. (2016), Hoteles. En: http:// hoteleshuatulco.com.mx (Consultado el 6 de junio de 2016).

Carrillo, Javier, Del río, Pablo y Könnolä, Totti (2009), Eco-innovation: when sustainability and competitiveness shake hands. Éditions Lavoisier, Francia.

Centro de Investigación de Recursos y Consumos Energéticos, CIRCE (2013), Fomento de la ecoinnovación empresarial en Aragón. En: http://ecoinnovacion. fcirce.es/ (Consultado el 14 de marzo de 2014).

Comisión Europea para el Medioambiente (2009), ECO-INNOVATION 20082013. Cuando se asocian la empresa y el medio ambiente.

Equipo Verde Huatulco, EVH (2014), Programas del destino.En: http:// www.evh.org.mx/(Consultado el 1 de junio de 2014).

Fondo Nacional de Fomento al Turismo, FONATUR (2012), Huatulco: Información General.En: http:// www.fonatur.gob.mx/es/proyectos desarrollos/huatulco/(Consultado el día 3 de septiembre de 2014).

Fundación Canaria Medioambiental (2011), Ecoeficiencia y ecoinnovación.

Hellström, Tomas (2006), Dimensions of Environmentally Sustainable Innovation: the Structure of EcoInnovation Concepts, Sustainable Development, Vol. 15 (3), 148-159.

Horbach, Jens; Rammer, Christian and Rennings, Klaus (2012), Determinants of eco-innovations by type of environmental impact: The role of regulatory push/pull, technology push and market pull, Ecological Economics, Vol. 78, 112122.

Kammerer, Daniel (2009), The effects of customer benefit and regulation on environmental product innovation. Empirical evidence from appliance manufacturers in
Germany,Ecological Economics, Vol. 68 (9), 2285-2295.

Kemp, René y Oltra, Vanesa (2011), Research Insights and Challenges on EcoInnovation Dynamics, Industry and Innovation, Vol. 18 (3), 249-253.

Kemp, René y Pearson, Peter (2008), Final report MEI project about measuring ecoinnovation. Project Title: Measuring eco-innovation. OECD.

Kemp, René y Pontoglio, S. (2007), Workshop conclusions about panel survey analysis of eco-innovation and definitions. MEI Project.

Measuring Eco-Innovation Project, MEI (2007), Workshop conclusions about panel survey analysis and definitions of eco-innovation.

Miret, Luis, Segarra, María y Peiró, Ángel (2011), How to rate Ecoinnovation? A tourism sector indicator's analysis, Tec Empresarial, Vol. 5(2), 15-25.

Organisation for Economic Co-Operation and Development, OECD (2011), Guidelines for Multinational Enterprises.En: http://mneguidelines. oecd.org(Consultado el 27 de febrero de 2014)

Reid, Alasdair y Miedzinski, Michal (2008), Eco-innovation. Final report for sectoral innovation watch. Technopolis Group, Bruselas.

Rennings, Klaus (2000), Redefining innovation, eco-innovation research and the contribution from ecological economics. Ecological Economics, Vol. 32 (2), 319-332.

Secretaría de Turismo y Desarrollo Económico del Estado de Oaxaca, STYDE (2014), Boletín Ejecutivo de la Actividad Turística en el Estado. En: http://www.styde.oaxaca.gob.mx/ indicadores.html (Consultado el día 28 de agosto, de 2014).

Valero, Antonio (2013), Fomento de la Ecoinnovación Empresarial. Encuentro de Eco-innovación Empresarial 2013. En: http://ecoinnovacion.fcirce. es/sites/default/files/innovacion/ docs/00 ANTONIO VALERO.pdf (Consultado el 15 de marzo de 2014). 
Elementos determinantes de la ecoinnovación en hotelería de Huatulco, México Judith Alejandra Velázquez-Castro, Elva Esther Vargas-Martínez

Velázquez, Judith y Vargas, Elva (2014), Ecoinnovación en turismo: una aproximación al estado de la cuestión, Gestión y Ambiente, Vol. 17(1), 191-207.
Wagner, Marcus (2008), Empirical influence of environmental management on innovation: evidence from Europe. Ecological Economics, Vol. 66 (3), 392-402. 


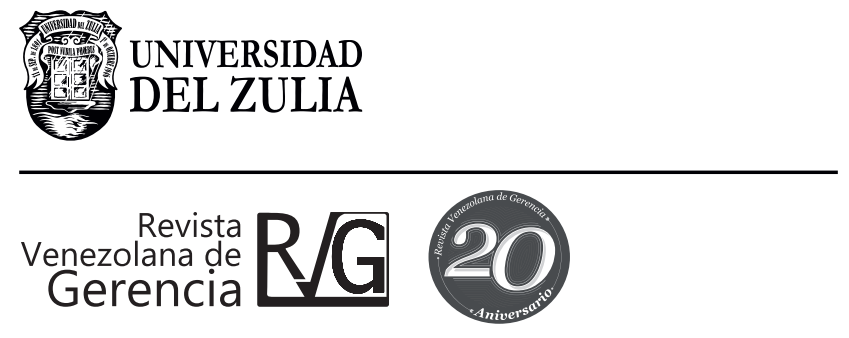

Año 21, No. 74

Esta revista fue editada en formato digital y publicada en junio del 2016, por la Universidad del Zulia, Vicerrectorado Académico, Serbiluz - Fondo editorial, Maracaibo -Venezuela. 